\title{
Reporting of Patient And Public Involvement (PPI) In Clinical Trials Published In Three Leading Nursing Science Journals: A Scientiometric Study
}

Richard Gray ( $\nabla$ r.gray@latrobe.edu.au )

La Trobe University - Bundoora Campus: La Trobe University https://orcid.org/0000-0001-9694-4206

Catherine Brasier

La Trobe University - Bundoora Campus: La Trobe University

Ashley $\mathrm{H} \mathrm{Ng}$

La Trobe University - Bundoora Campus: La Trobe University

\section{Research Article}

Keywords: Patient and public involvement, clinical trials, nursing

Posted Date: July 9th, 2021

DOI: https://doi.org/10.21203/rs.3.rs-665296/v1

License: () (i) This work is licensed under a Creative Commons Attribution 4.0 International License. Read Full License 


\section{Abstract}

Background: Patient and Public Involvement (PPI) in identifying research questions, designing trials, and collecting, interpreting, and publishing results may positively affect the relevance, quality and impact of research. The extent of patient and public involvement in nursing clinical trials has not been previously studied.

Methods: A Scientiometric study of PPI in clinical trials published in three leading nursing science journals. Randomised controlled clinical trials published in the International Journal of Nursing Studies, the Journal of Clinical Nursing, and the Journal of Advanced Nursing between January and April 2021 were identified by searching the SCOPUS database. Journal author guidelines were also review. Data were extracted against the five items of the Guidance for Reporting Involvement of Patients and the Public (GRIPP2) reporting checklist.

Results: None of the journals required authors to include a patient and public involvement statement in submitted papers or to thank participants in the acknowledgement section of the paper. We included 23 randomised controlled clinical trials, of which half were conducted in Europe and 12 were supported by external grants. Authors thanked participants in the acknowledgement section often (43\%) trials. There was no statement or evidence of patient and public involvement in any of the included trials.

Conclusion: There was no evidence of PPI in trials published in nursing science journals. Researchers, funders, and journals need to collaborate to change cultural expectations to ensure that PPI is an emended part of clinical research.

\section{Plain English Summary}

Patient involvement in research improves the quality and relevance of the work. The British Medical Journal - one of the top medical research journals in the World - has developed a patient partnership plan. Part of this plan is that papers sent to the journal for publication must include a statement about how patients were involved in the research. If patients were not involved in the research, this needed to be stated in the paper. Most papers published in the British Medical Journal now include a statement about patient involvement. We wanted to check if nurses were reporting patient involvement in the research they do. We read 23 randomised controlled trials (the type of research that is most likely to change what nurses do in hospitals) published in the top three nursing journals in the World. Unlike the British Medical Journals, there was no evidence that nursing Journals required authors to say how patients were involved in the research they published. There was no evidence of patient involvement in any of the nursing research articles that we looked at. The implication of our work is that the editors of nursing journals and nurse researchers need to be developing ways for meaningful engagement of patients in all aspects of their research and explaining how they did this in the papers they publish in nursing journals.

\section{Introduction}

Patient and public involvement (PPI) in research can be defined as research carried out 'with' or 'by' members of the public rather than 'to', 'about' or 'for' them (NIHR, n.d.). There is a clear consensus that patient and public involvement in research improves the relevance, quality, and impact of the work (Price, Albarqouni, et al., 2018; Vale et al., 2012). In PPI, consumers are active partners in all aspects of research, from generating the research question, writing the grant application, developing the study protocol, collecting data, analysing and interpreting results and co-authoring publications (Vale et al., 2012).

Major research funding bodies are, to a varying degree, encouraging of PPI. In the United Kingdom, for example, the National Institute for Health Research (NIHR) - the major funding body for health research - has taken a notably forthright position, requiring researchers applying for funding to "describe how they have involved the public in the design and planning of their study as well as their plans for further involvement throughout the research, including plans for evaluating impact" (NIHR, n.d.). In Australia National Health and Medical Research Council encourages researchers to involve patients in all stages of the research process (NHMRC, 2016).

While researchers are seemingly, generally optimistic about involving patients in research, they struggle to achieve this objective (Boaz et al., 2016). Authors of a qualitative exploration of 36 researchers' experiences of PPI, for example, found that participants expressed a combination of ambivalence, cynicism and enthusiasm about PPI (Boylan et al., 2019). Further, Boylan et al. (2019) report that participants described PPI as both rewarding and burdensome, requiring practical and social support. Bowers et al. (2020) argue that PPI in research is time-consuming and more challenging for patients, the public and researchers than is often argued.

We have identified four studies that have examined the extent to which PPI has been integrated into research (Jones et al., 2015; Owyang et al., 2021; Patel et al., 2021; Price, Schroter, et al., 2018). Owyang et al. (2021) report a systematic review examining the prevalence and quality of PPI in randomised controlled trials in orthopaedics. The authors reviewed 475 studies and identified two trials where there was some evidence of meaningful PPI. In one, PPI was used to inform the research question, choice of outcome and the dissemination of findings. In the second trial, PPI informed the study design.

The British Medical Journal (BMJ) has been influential in promoting PPI through its Patient Partnership Strategy. In 2014 the $B M J$ implemented a policy requiring authors to make a PPI deceleration in the methods section of submitted manuscripts. Authors that had not involved patients were asked to state this in the paper explicitly. This policy aimed to increase PPI in research by shifting cultural expectations. Price et al. (2018) report the frequency of PPI in research published in the $B M J$ before and after implementing the consumer involvement policy. Of 152 papers - reporting any study design - published in the year following the implementation of the policy, there was an increase from less than $1-11 \%$ of authors reporting PPI, an important improvement but suggesting that consumer involvement in research is still infrequent.

Staniszewska et al. (2017) discussed the development of the GRIPP2 (Guidance for Reporting Involvement of Patients and the Public version 2) checklist for reporting patient and public involvement in research. The authors used the EQUATOR method for developing reporting guidelines: a three-round Delphi study

Page $2 / 12$ 
and consensus statement. GRIPP2 identified key concepts that authors should report in their papers to describe PPI in their research. The short-form version of the guideline addresses five topics: a. the aim of PPI in the study, b. methods used for PPI, c. results of PPI in the study, d. extent to which PPI influenced the study overall, e. reflections. The authors suggest that journal editors can use the GRIPP2 checklist to set reporting expectations for submitted manuscripts.

The extent to which PPI is demonstrated in clinical trials in nursing has not been previously examined. We undertook a scientiometric study to show to what extent nurses involve consumers in randomised controlled clinical trials reported in nursing science journals.

\section{Methods}

\section{Pre-registration}

The methodology for this study was registered with the Open Science Framework and can be accessed via this link: https://osf.io/x2bqv

\section{Ethical considerations}

Ethical approval for this study was not required because data were extracted from publicly available sources or published research.

\section{Consumer academic involvement}

The study was instigated by RG, who does not identify as a consumer researcher. AN and CB are employed as teaching and research academics and identify as consumers. Both are committed to consumer and stakeholder engagement and were involved across all stages of this project.

\section{Study design}

We conducted a scientiometric study of PPI in randomised controlled clinical trials published between January and May 2021 in the leading nursing science journals.

\section{Joumal author guidelines}

Two researchers read the journal author guidelines - and any other supplementary documents - to identify any requirements to report patient and public involvement in submitted manuscripts. We also checked if authors were instructed to thank patients involved in studies in the acknowledgement section of the manuscript.

\section{Sample}

We identified a sample of papers reporting the results of randomised controlled clinical trials published in three leading (publish more than 200 documents per year) general nursing science Journals: the International Journal of Nursing Studies (IJNS), the Journal of Advanced Nursing (JAN) and the Journal of Clinical Nursing (JCN) from 1 January 2021 through 31 April 2021. Trials testing an educational intervention or did not involve a clinical population were excluded. Our decision to focus on randomised clinical trials was informed by the impact this high level of evidence would have on patient care and treatment. We also considered that it was more likely that randomised controlled trials were more likely to have received funding where PPI was a requirement.

\section{Search strategy}

All documents published in the IJNS, JAN and $J C N$, were identified using the "sources" function in SCOPUS, entering the journal title and accessing the "SCOPUS content coverage" tab. We then restricted documents to those published in 2021. Title and abstract and full-text screening of all documents published (including the early view) between 1 January 2021 and 31 April 2021 was completed by two researchers to determine those that met our inclusion criteria.

\section{Data extraction}

Included papers were read in full - including footnotes and acknowledgements - by two researchers who independently extracted the following information: citation, summary of the trial, country where fieldwork was conducted, if any authors identified as being a lived experience researcher, a summary of PPI reported in the manuscript, if participants were thanked in the acknowledgement section, source(s) of funding (coded: a. charity, b. government, c. University, d. own account, e. student project). Additionally, information against the five GRIPP2 short-form items was also extracted.

\section{PPI requirement of funding bodies}

We checked the website of organisations identified in included manuscripts as funding trials to determine if PPI was a recommendation or requirement of funding applications.

\section{Results}

\section{Joumal author guidelines}

The author guidelines of the three participating journals neither implicitly nor explicitly required authors to report how patients were involved in the research submitted to the journal. All three journals required that authors follow relevant reporting guidelines listed on the EQUATOR network but did not mention GRIPP or GRIPP2. The Journal of Clinical Nursing author guidelines does refer to two editorials about the position of the journal about reporting guidelines in 
qualitative research (Smith, Gelling, Haigh, Barnason, Allan, \& Jackson, 2018) and clinical trials specifically (Smith, Gelling, Haigh, Barnason, Allan, Penny, et al., 2018). In neither editorial is patient and public involvement mentioned. The author guidelines do not indicate if patients can be included as authors in submitted papers.

Both the Journal of Clinical Nursing and the Journal of Advanced Nursing has identical text about what information authors should include in the acknowledgement section and make no mention of thanking study participants. Section 2.9 of the author guidelines for the International Journal of Nursing Studies is the only section that deals with acknowledgements but is entirely focused on authorship.

\section{Included studies}

Figure 1 shows the flow of papers through the study. As of the end of April 2021, the IJNS, JCN and JANhad published 691 documents, of which 23 (3\%) were papers reporting the results of a randomised controlled clinical trial. A complete list of included trials has been made available via an online data repository that can be accessed via this link: (https://doi.org/10.26181/60bf1eac7eb64).

Table 1 summarises the information extracted from the included studies. Almost half of the corresponding authors were based in Europe $(\mathrm{n}=11,48 \%)$ most studies were focused on medical patients $(n=13,57 \%)$, half $(n=12,52 \%)$ were funded by an external charity $(n=4,17 \%)$, government $(n=5,22 \%)$ or University $(n=3,13 \%)$ grant. 
Table 1

, patient and public involvement in reports of results of clinical trials using GRIPP 2-SF

\begin{tabular}{|c|c|c|c|c|c|c|c|c|}
\hline & & & & & & & \multicolumn{2}{|c|}{ GRIPP 2 - short fo } \\
\hline Citation & Summary of trial & $\begin{array}{l}\text { Country where } \\
\text { corresponding } \\
\text { authors was } \\
\text { based }\end{array}$ & $\begin{array}{l}\text { Summary } \\
\text { of PPI in } \\
\text { the trial }\end{array}$ & $\begin{array}{l}\text { Participants were } \\
\text { thanked in the } \\
\text { acknowledgement } \\
\text { section of the } \\
\text { manuscript }\end{array}$ & Funding information & $\begin{array}{l}\text { At least } \\
\text { one of the } \\
\text { authors } \\
\text { Identifies } \\
\text { as having } \\
\text { lived } \\
\text { experience } \\
\text { (as a } \\
\text { patient) }\end{array}$ & $\begin{array}{l}\text { 1. Aim } \\
\text { (the aim } \\
\text { of PPI } \\
\text { in the } \\
\text { study }\end{array}$ & $\begin{array}{l}\text { 2. Meth } \\
\text { (descrif } \\
\text { of the } \\
\text { methoc } \\
\text { used fo } \\
\text { PPI in t } \\
\text { study) }\end{array}$ \\
\hline $\begin{array}{l}\text { Hassan et al } \\
(2021)\end{array}$ & $\begin{array}{l}\text { RCT testing the } \\
\text { Trendelenburg } \\
\text { position with } \\
\text { comparator in } \\
\text { obese critically ill } \\
\text { patients. The } \\
\text { experimental } \\
\text { position led to } \\
\text { improved } \\
\text { respiratory } \\
\text { parameters in } \\
110 \text { participants }\end{array}$ & Egypt & None & Yes & Not reported & No & $\begin{array}{l}\text { Not } \\
\text { reported }\end{array}$ & $\begin{array}{l}\text { Not } \\
\text { reporte }\end{array}$ \\
\hline $\begin{array}{l}\text { Valeberg et } \\
\text { al (2021 }\end{array}$ & $\begin{array}{l}\text { Effect on pain of } \\
\text { a } \\
\text { psychoeducation } \\
\text { intervention to } \\
\text { improvement } \\
\text { pain } \\
\text { management } \\
\text { following day } \\
\text { surgery in } 220 \\
\text { participants }\end{array}$ & Norway & None & No & Not reported & No & $\begin{array}{l}\text { Not } \\
\text { reported }\end{array}$ & $\begin{array}{l}\text { Not } \\
\text { reporte }\end{array}$ \\
\hline $\begin{array}{l}\text { Østergaard } \\
\text { et al (2021) }\end{array}$ & $\begin{array}{l}\text { A RCT to test. } \\
\text { Family nursing } \\
\text { therapeutic } \\
\text { conversations in } \\
468 \text { people with } \\
\text { heart failure and } \\
\text { their family. } \\
\text { Social support } \\
\text { improved in the } \\
\text { intervention } \\
\text { compared to the } \\
\text { control. }\end{array}$ & Denmark & None & Yes & $\begin{array}{l}\text { The Health Foundation, } \\
\text { Danish Heart Foundation, } \\
\text { Novo Nordisk Foundation. } \\
\text { Could not find evidence that } \\
\text { PPI was a requirement of } \\
\text { recommendation of funding }\end{array}$ & No & $\begin{array}{l}\text { Not } \\
\text { reported }\end{array}$ & $\begin{array}{l}\text { Not } \\
\text { reporte }\end{array}$ \\
\hline $\begin{array}{l}\text { Simonetti et } \\
\text { al (2021) }\end{array}$ & $\begin{array}{l}\text { Training in self- } \\
\text { monitoring of } \\
\text { blood pressure } \\
\text { compared with } \\
\text { TAU in people } \\
\text { with } \\
\text { hypertension } \\
\text { improved patient } \\
\text { adherence to } \\
\text { treatment }\end{array}$ & Italy & None & No & Own account & No & $\begin{array}{l}\text { Not } \\
\text { reported }\end{array}$ & $\begin{array}{l}\text { Not } \\
\text { reporte }\end{array}$ \\
\hline $\begin{array}{l}\text { Zhao et al } \\
(2021)\end{array}$ & $\begin{array}{l}\text { RCT of a mixed } \\
\text { management } \\
\text { intervention in } \\
182 \text { prenatal } \\
\text { women } \\
\text { improved overall } \\
\text { and exclusive } \\
\text { breastfeeding }\end{array}$ & China & None & Yes & University funding & No & $\begin{array}{l}\text { Not } \\
\text { reported }\end{array}$ & $\begin{array}{l}\text { Not } \\
\text { reporte }\end{array}$ \\
\hline $\begin{array}{l}\text { Dincer and } \\
\text { Yildrim } \\
(2021)\end{array}$ & $\begin{array}{l}\text { To reduce pain } \\
\text { associated with } \\
\text { injection } 84 \\
\text { patients } \\
\text { attending the } \\
\text { emergency } \\
\text { department were } \\
\text { randomised to } \\
\text { receive vibration } \\
\text { stimulation or } \\
\text { treatment as } \\
\text { usual }\end{array}$ & Turkey & None & Yes & Own account & No & $\begin{array}{l}\text { Not } \\
\text { reported }\end{array}$ & $\begin{array}{l}\text { Not } \\
\text { reporte }\end{array}$ \\
\hline
\end{tabular}




\begin{tabular}{|c|c|c|c|c|c|c|c|c|}
\hline & & & & & & & \multicolumn{2}{|c|}{ GRIPP 2 - short fo } \\
\hline $\begin{array}{l}\text { Sahebkar et } \\
\text { al (2021) }\end{array}$ & $\begin{array}{l}\text { RCT testing } \\
\text { different } \\
\text { injection sites in } \\
\text { patients } \\
\text { attending the } \\
\text { emergency } \\
\text { department. } \\
\text { Choosing an } \\
\text { injection site } \\
\text { based on body } \\
\text { shape pattern } \\
\text { was associated } \\
\text { with lower levels } \\
\text { of reported pain }\end{array}$ & Iran & None & Yes & Master's degree project & No & $\begin{array}{l}\text { Not } \\
\text { reported }\end{array}$ & $\begin{array}{l}\text { Not } \\
\text { reporte }\end{array}$ \\
\hline $\begin{array}{l}\text { Basak et al } \\
(2021)\end{array}$ & $\begin{array}{l}91 \text { patients } \\
\text { prescribed } \\
\text { intramuscular } \\
\text { antibiotic } \\
\text { medication were } \\
\text { randomised to } \\
\text { virtual reality, } \\
\text { distraction cards } \\
\text { or control } \\
\text { conditions. Pain } \\
\text { levels were lower } \\
\text { in both virtual } \\
\text { reality and } \\
\text { distraction card } \\
\text { conditions } \\
\text { compared to } \\
\text { control. }\end{array}$ & Turkey & None & Yes & Not reported & & $\begin{array}{l}\text { Not } \\
\text { reported }\end{array}$ & $\begin{array}{l}\text { Not } \\
\text { reporte }\end{array}$ \\
\hline $\begin{array}{l}\text { Liebergall- } \\
\text { Wischnitzer } \\
\text { et al. (2021) }\end{array}$ & $\begin{array}{l}64 \text { women } \\
\text { following } \\
\text { elective } \\
\text { caesarean } \\
\text { section } \\
\text { randomised to } \\
\text { receive Paula } \\
\text { method } \\
\text { exercises or } \\
\text { treatment as } \\
\text { usual. The } \\
\text { exercises were } \\
\text { effective at } \\
\text { promoting the } \\
\text { resumption of } \\
\text { gastrointestinal } \\
\text { activity }\end{array}$ & Israel & None & No & $\begin{array}{l}\text { Not reported in the } \\
\text { manuscript }\end{array}$ & $\begin{array}{l}\text { Job titles } \\
\text { of authors } \\
\text { not } \\
\text { reported }\end{array}$ & $\begin{array}{l}\text { Not } \\
\text { reported }\end{array}$ & $\begin{array}{l}\text { Not } \\
\text { reporte }\end{array}$ \\
\hline $\begin{array}{l}\text { Zhang et al } \\
(2021)\end{array}$ & $\begin{array}{l}\text { Caregivers }(\mathrm{n}= \\
125) \text { of patients } \\
\text { with permanent } \\
\text { enterostomy } \\
\text { randomised to } \\
\text { receive either the } \\
\text { hospital family } \\
\text { holistic care or } \\
\text { TAU. The } \\
\text { experimental } \\
\text { intervention led } \\
\text { to improved care } \\
\text { ability of care } \\
\text { givers }\end{array}$ & China & None & Yes & $\begin{array}{l}\text { Dreyfus Health Founding, } \\
\text { Fujian Provincial Health } \\
\text { Technology Project, and } \\
\text { National Key Clinical } \\
\text { Specialty Discipline } \\
\text { Construction Program of } \\
\text { China }\end{array}$ & $\begin{array}{l}\text { Job titles } \\
\text { of authors } \\
\text { not } \\
\text { reported }\end{array}$ & $\begin{array}{l}\text { Not } \\
\text { reported }\end{array}$ & $\begin{array}{l}\text { Not } \\
\text { reporte }\end{array}$ \\
\hline $\begin{array}{l}\text { Milazi et al } \\
(2021)\end{array}$ & $\begin{array}{l}\text { Self- } \\
\text { management of } \\
\text { was more } \\
\text { effective than } \\
\text { treatment as } \\
\text { usual in helping } \\
\text { patients with } \\
\text { end-stage kidney } \\
\text { disease achieve } \\
\text { phosphate } \\
\text { control }\end{array}$ & Australia & None & Yes & $\begin{array}{l}\text { Royal Brisbane and Women's } \\
\text { Hospital Research } \\
\text { Foundation, Health and } \\
\text { Medical Research Unit, } \\
\text { Queensland Health, Nursing } \\
\text { and Midwifery Research } \\
\text { Fellowship and The } \\
\text { Queensland Nurses and } \\
\text { Midwives' Union. No evidence } \\
\text { PPI was a condition of } \\
\text { funding }\end{array}$ & $\begin{array}{l}\text { Job titles } \\
\text { of authors } \\
\text { not } \\
\text { reported }\end{array}$ & $\begin{array}{l}\text { Not } \\
\text { reported }\end{array}$ & $\begin{array}{l}\text { Not } \\
\text { reporte }\end{array}$ \\
\hline
\end{tabular}




\begin{tabular}{|c|c|c|c|c|c|c|c|c|}
\hline \multirow[b]{2}{*}{$\begin{array}{l}\text { Simón-López } \\
\text { et al (2021) }\end{array}$} & \multirow[b]{2}{*}{$\begin{array}{l}\text { Three arm } \\
\text { randomised } \\
\text { cross over trial } \\
\text { comparing heat, } \\
\text { pressure, heat } \\
\text { and pressure, } \\
\text { against control } \\
\text { in } 62 \text { healthy } \\
\text { volunteers. The } \\
\text { primary outcome } \\
\text { was successful } \\
\text { venous } \\
\text { catherization. At } \\
\text { the first attempt. } \\
\text { High pressure } \\
\text { was the most } \\
\text { effective } \\
\text { intervention }\end{array}$} & \multirow[b]{2}{*}{ Spain } & \multirow[b]{2}{*}{ None } & \multirow[b]{2}{*}{ No } & \multirow[b]{2}{*}{ Own account } & \multirow[b]{2}{*}{$\begin{array}{l}\text { Job titles } \\
\text { of authors } \\
\text { not } \\
\text { reported }\end{array}$} & \multicolumn{2}{|c|}{ GRIPP 2 - short fo } \\
\hline & & & & & & & $\begin{array}{l}\text { Not } \\
\text { reported }\end{array}$ & $\begin{array}{l}\text { Not } \\
\text { reporte }\end{array}$ \\
\hline $\begin{array}{l}\text { Riera- } \\
\text { Sampol et al } \\
(2021)\end{array}$ & $\begin{array}{l}\text { The } \\
\text { effectiveness of } \\
\text { nurse delivered } \\
\text { motivational } \\
\text { interviewing and } \\
\text { exercise } \\
\text { prescription } \\
\text { compared with } \\
\text { TAU in } 263 \text { with } \\
\text { at least two } \\
\text { cardiovascular } \\
\text { risk factors }\end{array}$ & Spain & None & No & $\begin{array}{l}\text { Fondo de Investigaciones } \\
\text { Sanitarias of Instituto de } \\
\text { Salud Carlos III. Could not } \\
\text { identify any evidence that PPI } \\
\text { was a } \\
\text { requirement/recommendation } \\
\text { of funding }\end{array}$ & $\begin{array}{l}\text { Job titles } \\
\text { of authors } \\
\text { not } \\
\text { reported }\end{array}$ & $\begin{array}{l}\text { Not } \\
\text { reported }\end{array}$ & $\begin{array}{l}\text { Not } \\
\text { reporte }\end{array}$ \\
\hline $\begin{array}{l}\text { de Pinho et } \\
\text { al (2021) }\end{array}$ & $\begin{array}{l}\text { Metacognitive } \\
\text { training for } 56 \\
\text { people with } \\
\text { schizophrenia } \\
\text { was effective at } \\
\text { reducing } \\
\text { psychotic } \\
\text { symptoms } \\
\text { compared with } \\
\text { TAU. }\end{array}$ & Portugal & None & No & $\begin{array}{l}\text { The Portuguese Society of } \\
\text { Mental Health Nursing } \\
\text { (ASPESM) and CINTESIS } \\
\text { R\&D Unit. Could not identify } \\
\text { any evidence that PPI was a } \\
\text { requirement/recommendation } \\
\text { of funding. }\end{array}$ & $\begin{array}{l}\text { Job titles } \\
\text { of authors } \\
\text { not } \\
\text { reported }\end{array}$ & $\begin{array}{l}\text { Not } \\
\text { reported }\end{array}$ & $\begin{array}{l}\text { Not } \\
\text { reporte }\end{array}$ \\
\hline $\begin{array}{l}\text { Tamayo- } \\
\text { Morales et al } \\
(2021)\end{array}$ & $\begin{array}{l}\text { A RCT of a } \\
\text { behavioural } \\
\text { intervention was } \\
\text { effective at } \\
\text { reducing } \\
\text { behavioural } \\
\text { disturbance in } \\
\text { older adults } \\
\text { attending day } \\
\text { care centres }\end{array}$ & Spain & None & No & $\begin{array}{l}\text { Three funding bodies: a. } \\
\text { Carlos III Health Institute of } \\
\text { the Ministry of Science and } \\
\text { Innovation, b. European } \\
\text { Development Funds, c. } \\
\text { Autonomous Government of } \\
\text { Castilla y Leon). Could not } \\
\text { find any evidence that PPI } \\
\text { was a } \\
\text { requirement/recommendation } \\
\text { of funder. }\end{array}$ & $\begin{array}{l}\text { Job titles } \\
\text { of authors } \\
\text { not } \\
\text { reported }\end{array}$ & $\begin{array}{l}\text { Not } \\
\text { reported }\end{array}$ & $\begin{array}{l}\text { Not } \\
\text { reporte }\end{array}$ \\
\hline $\begin{array}{l}\text { Tonye- } \\
\text { Geoffroy et } \\
\text { al (2021) }\end{array}$ & $\begin{array}{l}\text { Hypnosis for } \\
\text { chronic pain was } \\
\text { tested in } 72 \\
\text { patients } \\
\text { receiving } \\
\text { treatment with } \\
\text { TENS. No } \\
\text { difference } \\
\text { between the } \\
\text { groups were } \\
\text { observed }\end{array}$ & France & None & No & $\begin{array}{l}\text { Paramedicale Nursing } \\
\text { Research Hospital Program. } \\
\text { Website in French only }\end{array}$ & $\begin{array}{l}\text { Job titles } \\
\text { of authors } \\
\text { not } \\
\text { reported }\end{array}$ & $\begin{array}{l}\text { Not } \\
\text { reported }\end{array}$ & $\begin{array}{l}\text { Not } \\
\text { reporte }\end{array}$ \\
\hline $\begin{array}{l}\text { Bunsanong } \\
\text { and } \\
\text { Chaimongkol } \\
(2021)\end{array}$ & $\begin{array}{l}\text { RCT of a self- } \\
\text { management } \\
\text { intervention for } \\
\text { "middle-aged" } \\
\text { women with } \\
\text { knee } \\
\text { osteoarthritis. } \\
\text { Self- } \\
\text { management } \\
\text { resulted in better } \\
\text { knee functional } \\
\text { status compared } \\
\text { to TAU }\end{array}$ & Thailand & None & Yes & University funding & $\begin{array}{l}\text { Job titles } \\
\text { of authors } \\
\text { not } \\
\text { reported }\end{array}$ & $\begin{array}{l}\text { Not } \\
\text { reported }\end{array}$ & $\begin{array}{l}\text { Not } \\
\text { reporte }\end{array}$ \\
\hline
\end{tabular}




\begin{tabular}{|c|c|c|c|c|c|c|c|c|}
\hline & & & & & & & \multicolumn{2}{|c|}{ GRIPP 2 - short fo } \\
\hline $\begin{array}{l}\text { Morales- } \\
\text { Fernández et } \\
\text { al (2021) }\end{array}$ & $\begin{array}{l}\text { Nurse facilitated } \\
\text { intervention } \\
\text { (healthy } \\
\text { lifestyles, } \\
\text { education on } \\
\text { self-esteem, pain } \\
\text { awareness, } \\
\text { communication, } \\
\text { and relaxation) } \\
\text { compared to } \\
\text { TAU in } 279 \\
\text { patients with } \\
\text { chronic } \\
\text { malignant pain. }\end{array}$ & Spain & None & No & Government & $\begin{array}{l}\text { Job titles } \\
\text { of authors } \\
\text { not } \\
\text { reported }\end{array}$ & $\begin{array}{l}\text { Not } \\
\text { reported }\end{array}$ & $\begin{array}{l}\text { Not } \\
\text { reporte }\end{array}$ \\
\hline $\begin{array}{l}\text { Šümen and } \\
\text { Öncel (2021) }\end{array}$ & $\begin{array}{l}\text { A three arm RCT } \\
\text { involving } 185 \\
\text { parents testing } \\
\text { education } \\
\text { against } \\
\text { education and } \\
\text { text messaging } \\
\text { and control. The } \\
\text { "I am protecting } \\
\text { my child from } \\
\text { the sun" plus text } \\
\text { messaging was } \\
\text { effective at } \\
\text { changing } \\
\text { parents' sun } \\
\text { protection } \\
\text { behaviour }\end{array}$ & Turkey & None & No & $\begin{array}{l}\text { Akdeniz University Scientific } \\
\text { Research Projects } \\
\text { Coordination Unit (could not } \\
\text { determine if PPI was a } \\
\text { requirement of funder as } \\
\text { could not access the website) }\end{array}$ & $\begin{array}{l}\text { Job titles } \\
\text { of authors } \\
\text { not } \\
\text { reported }\end{array}$ & $\begin{array}{l}\text { Not } \\
\text { reported }\end{array}$ & $\begin{array}{l}\text { Not } \\
\text { reporte }\end{array}$ \\
\hline $\begin{array}{l}\text { Shen et al } \\
(2021)\end{array}$ & $\begin{array}{l}\text { One-hundred } \\
\text { patients with } \\
\text { COPD } \\
\text { randomised to } \\
\text { active cycle } \\
\text { breathing } \\
\text { technique or } \\
\text { TAU. Sputum } \\
\text { production - the } \\
\text { primary outcome } \\
\text { - increased in } \\
\text { the experimental } \\
\text { compared to } \\
\text { control } \\
\text { intervention }\end{array}$ & China & None & No & Own account & $\begin{array}{l}\text { Job titles } \\
\text { of authors } \\
\text { not } \\
\text { reported }\end{array}$ & $\begin{array}{l}\text { Not } \\
\text { reported }\end{array}$ & $\begin{array}{l}\text { Not } \\
\text { reporte }\end{array}$ \\
\hline $\begin{array}{l}\text { Chew et al } \\
\text { (2021) }\end{array}$ & $\begin{array}{l}\text { Push-pull-hold } \\
\text { program } \\
\text { compared to } \\
\text { TAU in } 144 \text { heart } \\
\text { failure patients. } \\
\text { Self-care } \\
\text { improved in the } \\
\text { experimental } \\
\text { compared to the } \\
\text { TAU group. }\end{array}$ & Singapore & None & No & PhD scholarship & $\begin{array}{l}\text { Job titles } \\
\text { of authors } \\
\text { not } \\
\text { reported }\end{array}$ & $\begin{array}{l}\text { Not } \\
\text { reported }\end{array}$ & $\begin{array}{l}\text { Not } \\
\text { reporte }\end{array}$ \\
\hline $\begin{array}{l}\text { Lescop et al } \\
(2021)\end{array}$ & $\begin{array}{l}\text { Non-inferiority } \\
\text { trial testing the } \\
\text { Buzzy device } \\
\text { with lidocaine } \\
\text { path in children } \\
\text { undergoing a } \\
\text { needle-related } \\
\text { procedure. A } \\
\text { total of } 219 \\
\text { children were } \\
\text { randomised. } \\
\text { Pain levels were } \\
\text { lower in the } \\
\text { comparator } \\
\text { compared to the } \\
\text { Buzzy device } \\
\text { group. }\end{array}$ & France & None & Yes & $\begin{array}{l}\text { French Ministry of Health. } \\
\text { Could not identify the funding } \\
\text { scheme from the information } \\
\text { provided by the authors in the } \\
\text { manuscript. }\end{array}$ & $\begin{array}{l}\text { Job titles } \\
\text { of authors } \\
\text { not } \\
\text { reported }\end{array}$ & $\begin{array}{l}\text { Not } \\
\text { reported }\end{array}$ & $\begin{array}{l}\text { Not } \\
\text { reporte }\end{array}$ \\
\hline
\end{tabular}




\begin{tabular}{|c|c|c|c|c|c|c|c|c|}
\hline & & & & & & & \multicolumn{2}{|c|}{ GRIPP 2 - short fo } \\
\hline $\begin{array}{l}\text { Nesset et al } \\
(2021)\end{array}$ & $\begin{array}{l}\text { RCT comparing } \\
\text { cognitive } \\
\text { behaviour } \\
\text { therapy (CBT) or } \\
\text { mindfulness in } \\
125 \text { men that } \\
\text { had perpetrated } \\
\text { intimate partner } \\
\text { violence. No } \\
\text { difference } \\
\text { against the } \\
\text { primary outcome } \\
\text { - anxiety and } \\
\text { depression - } \\
\text { was reported } \\
\text { between the } \\
\text { groups }\end{array}$ & Norway & None & No & Own account & $\begin{array}{l}\text { Job titles } \\
\text { of authors } \\
\text { not } \\
\text { reported }\end{array}$ & $\begin{array}{l}\text { Not } \\
\text { reported }\end{array}$ & $\begin{array}{l}\text { Not } \\
\text { reporte }\end{array}$ \\
\hline
\end{tabular}

TAU = Treatment as usual, COPE = Chronic Obstructive Pulmonary Disease

Patient and public involvement

None of the researchers listed as authors on included studies identified as lived experience or patient researchers.

Authors of most $(n=13,57 \%)$ of the included studies did not thank participants for their contribution to the research in the acknowledgement section of the manuscript.

A summary of patient and public involvement was not reported in any of the included manuscripts. There was no evidence of patient or public involvement in any of the included studies; consequently, we could not rate any of the GRIPP2 short-form items.

\section{PPI requirements of funding bodies}

We searched the websites of 16 funding bodies that had supported included but could not find any evidence that PPI was recommended or a requirement of funding being awarded. All of the websites had an English language version except two that were exclusively in French.

\section{Discussion}

This study aimed to investigate the reporting of patient and public involvement in clinical trials published in the leading journals in nursing science. We found no evidence of PPI in any of the 23 trials that we reviewed. Our finding is consistent with similar studies in other clinical disciplines (Jones et al., 2015; Owyang et al., 2021; Patel et al., 2021). For example, Owyang et al. (2021) found evidence of patient and public involvement in only two of the 475 orthopaedic trials they included in their review. Notably, much higher rates of PPI have been reported in the British Medical Journal, where 1 in 10 studies have some form of PPI activity (Price, Schroter, et al., 2018).

None of the three nursing science journals we included in this review required authors to report PPI in the papers they publish. Again, authors of studies in other disciplines reported similar findings. For example, in the study of PPI in orthodontic research, Patel et al. (2021) reported that none of the four journals that they included had guidance on PPI. The exception is the British Medical Journal that actively promotes PPI in papers they publish. The BMJ introduced a policy in 2014 requiring authors submitting manuscripts to the journal to include a PPI declaration in their methods section (Price, Schroter, et al., 2018).

Trials published in nursing science journals were seemingly not supported by funding bodies that required PPI as part of the grant application process. Our observation is somewhat discrepant with other disciplines; for example, Patel et al. (2021) reported in their study of PPI that of nine funding agencies that they reviewed, only two (the National Institute for Health Research and Health Care Research Wales) required applicants to demonstrate PPI as part of the application process.

It is part of the $B M J$ patient partner strategy that authors should thank the patients that participated in the research in the acknowledgement section of their manuscript (Price, Schroter, et al., 2018). Expressing gratitude to people giving freely of their time in the advancement of knowledge seems to us important. It is the least that researchers can do, particularly as published research articles are often inaccessible to consumers as they are either behind a paywall or jargon-heavy and challenging for the general public to understand. That over half of the included nursing trials did not thank patients for their contribution is disappointing.

Limitations

It may be that researchers are involving patients in their research and then not reporting this in the published manuscript. In their study of PPI rates, Patel et al. (2021) attempted to check - by emailing authors - if there was PPI in the trial, but this had not been reported in the manuscript. Only $14 \%$ responded to the researcher's request, suggesting that directly contacting authors may not be an effective way to check unreported PPI (Patel et al., 2021). Based on this observation, we concluded that there was little merit in contacting authors to check unreported PPI. It may be that qualitative interviews with researchers may be a more productive approach to addressing this issue.

Identifying if authors of included studies were consumer researchers was problematic. We assumed that it would be evident in the author affiliation if this were the case. However, none of the three included journals asks authors to report job titles in papers, and this is probably how we would most likely identify 
lived experience authors. We also note that there is a culture in academia not to identify as lived experience academics. So even though authors may have an academic affiliation, they may still be a lived experience academic, but we would not know.

We note that it was difficult to determine if some funding bodies required PPI because different terms were used, also some of the websites were not in English, making it difficult for us to be confident that PPI was not a requirement of funding.

Generalisability

The generalisability of our observation is limited by the small number of journals that we included and our decision to only focus on papers reporting clinical trials. We included three leading nursing journals that publish - between them - around 1,000 papers a year and are all ranked in the top 25 nursing science journals (Journal Citation Report) Impact Factor. It may be that there are higher levels of PPI reported in papers in more specialist nursing journals. For example, we are aware that the Journal of Psychiatric and Mental Health Nursing has a specific section on lived experience narratives. We focused on clinical trials because this design because is likely to have a direct impact on patient care, making PPI, arguably, more important. It may be that PPI is more prevalent in observational and qualitative research and systematic reviews.

Nurses may publish their research in non-nursing journals because they may perceive them as more prestigious or subject relevant. It is plausible that these studies are more likely to report meaningful PPI engagement to those in nursing journals. Consequently, we cannot generalise our observations to all trials by nurses, instead to those published in nursing science journals.

\section{Consumer academic perspectives (NG CB) on the study findings}

The original premise and intent of PPI in research is to ensure that the resulting work benefits the community it serves. Partnerships with various stakeholders in research are critical to co-design and develop solutions that are more likely to be implemented and used within practice. Patients and the general public need to be seen a stakeholder as important as clinicians and policymakers who are often included in research design and implementation.

Results from this study highlight that while there is a slow culture change to acknowledge patients and the public as important equals in research, there is still much to be done across journals and research institutions alike. It is not surprising that researchers often focus on the barriers when it comes to PPI in research, which is perhaps the next knot to untangle in embracing PPI.

\section{Consumer academic (NG, $\mathrm{CB}$ ) experiences of involving patients and the public in research}

Including PPI in research provides a different perspective from researchers and clinicians when it comes to tackling issues in healthcare. Patients and the public are privy to the challenges they face when navigating the healthcare system that may be unbeknownst or considered by researchers or clinicians.

However, it can be challenging to find patients and the public who can speak freely amongst the research team, particularly when hierarchical systems are in place. Additionally, while PPI can be embedded in research grants, it can sometimes be done in a tokenistic manner, diminishing the value of true PPI and increasing cynicism in patients to be involved with future projects.

\section{Conclusions}

Despite agreement about the value of PPI in improving the relevance and impact of research, we found no evidence that nurses had meaningfully engaged consumers in clinical trials. Further, authors frequently did not thank patients for their contribution to the research in the acknowledgement section of their manuscripts. Unlike their medical counterparts, the editorial leading nursing journals have not implemented policies to promote patient engagement in research. Researchers, funding agencies and Journals need to engage in strategies to promote meaningful PPI across all aspects of the research process.

\section{Abbreviations}

PPI: Patient and Public Involvement; BMJ British Medical Journal; GRIPP2 Guidance for Reporting Involvement of Patients and the Public; National Institute for Health Research (NIHR); National Health and Medical Research Council (NHMRC); Journal of Advanced Nursing (JAN); Journal of Clinical Nursing (JCN); International Journal of Nursing Studies (IJNS);

\section{Declarations}

\section{Acknowledgements}

Not applicable

\section{Transparency statement}

The Lead author affirms that the manuscript is an honest, accurate, and transparent account of the study being reported, that no important aspects of the study have been omitted; and that any discrepancies from the study as planned (and, if relevant, registered) have been explained.

\section{Dissemination statement}

Following the publication of this paper, we will communicate the results of the work via Twitter and Linkedln. Additionally, we will send a copy of the paper to the editors of the included journals. 


\section{Authors' contributions}

Conception and design RG, CB and AN. Data extraction and analysis RG, CB and AN. RG wrote a first draft of the manuscript. CB and AN edited and contributed to revisions of the paper.

\section{Funding}

An own account project, no external funding.

\section{Availability of data and materials}

A list of included studies is available via the link in the main body of the manuscript.

\section{Ethics approval and consent to participate}

Ethical approval for this study was not required.

\section{Consent for publication}

Not applicable

\section{Competing interest}

The authors declare no conflicts of interest.

\section{Author Details}

1. The School of Nursing and Midwifery, La Trobe University, Melbourne, VIC 3086, Australia.

2. Department of Social Work and Social Policy, La Trobe University, Melbourne, VIC 3086, Australia.

3. Department of Dietetics, Human Nutrition and Sport, La Trobe University, Melbourne, VIC 3086, Australia.

\section{References}

1. Boaz A, Biri D, McKevitt C. Rethinking the relationship between science and society: Has there been a shift in attitudes to Patient and Public Involvement and Public Engagement in Science in the United Kingdom? Health Expect. 2016;19(3):592-601. https://doi.org/10.1111/hex.12295.

2. Bowers B, Lovick R, Pollock K, Barclay S. Patient and public involvement in general practice research. Br J Gen Pract. 2020;70(694):220-1.

3. Boylan A-M, Locock L, Thomson R, Staniszewska S. About sixty per cent I want to do it”: Health researchers' attitudes to, and experiences of, patient and public involvement (PPI)-A qualitative interview study. Health Expect. 2019;22(4):721-30. https://doi.org/10.1111/hex.12883.

4. Briefing notes for researchers-Public involvement in NHS, health and social care research. (n.d.). Retrieved 16 June 2021, from https://www.nihr.ac.uk/documents/briefing-notes-for-researchers-public-involvement-in-nhs-health-and-social-care-research/27371.

5. Jones EL, Williams-Yesson BA, Hackett RC, Staniszewska SH, Evans D, Francis NK. Quality of reporting on patient and public involvement within surgical research: A systematic review. Ann Surg. 2015;261(2):243-50. https://doi.org/10.1097/SLA.0000000000000768.

6. Owyang D, Bakhsh A, Brewer D, Boughton OR, Cobb JP. (2021). Patient and Public Involvement Within Orthopaedic Research: A Systematic Review. JBJS, Latest Articles, 10.2106/JBJS.20.01573. https://doi.org/10.2106/JBJS.20.01573.

7. Patel VA, Shelswell J, Hillyard N, Pavitt S, Barber SK. A study of the reporting of patient and public involvement and engagement (PPIE) in orthodontic research. Journal of Orthodontics. 2021;48(1):42-51. https://doi.org/10.1177/1465312520968574.

8. Price A, Albarqouni L, Kirkpatrick J, Clarke M, Liew SM, Roberts N, Burls A. Patient and public involvement in the design of clinical trials: An overview of systematic reviews. J Eval Clin Pract. 2018;24(1):240-53. https://doi.org/10.1111/jep.12805.

9. Price A, Schroter S, Snow R, Hicks M, Harmston R, Staniszewska S, Parker S, Richards T. Frequency of reporting on patient and public involvement (PPI) in research studies published in a general medical journal: A descriptive study. BMJ Open. 2018;8(3):e020452. https://doi.org/10.1136/bmjopen-2017020452.

10. Smith GD, Gelling L, Haigh C, Barnason S, Allan H, Jackson D. The position of reporting guidelines in qualitative nursing research. J Clin Nurs. 2018;27(56):889-91. https://doi.org/10.1111/jocn.14213.

11. Smith GD, Gelling L, Haigh C, Barnason S, Allan H, Penny K, Jackson D. Transparency in the reporting of nursing research. J Clin Nurs. 2018;27(3-4):4757. https://doi.org/10.1111/jocn.14212.

12. Staniszewska S, Brett J, Simera I, Seers K, Mockford C, Goodlad S, Altman DG, Moher D, Barber R, Denegri S, Entwistle A, Littlejohns P, Morris C, Suleman R, Thomas V, Tysall C. GRIPP2 reporting checklists: Tools to improve reporting of patient and public involvement in research. BMJ. 2017;358:j3453. https://doi.org/10.1136/bmj.j3453

13. Vale CL, Thompson LC, Murphy C, Forcat S, Hanley B. Involvement of consumers in studies run by the Medical Research Council Clinical Trials Unit: Results of a survey. Trials. 2012;13:9. https://doi.org/10.1186/1745-6215-13-9.

\section{Figures}


SCOPUS

January 2021 to May 2021

691 Citation(s)

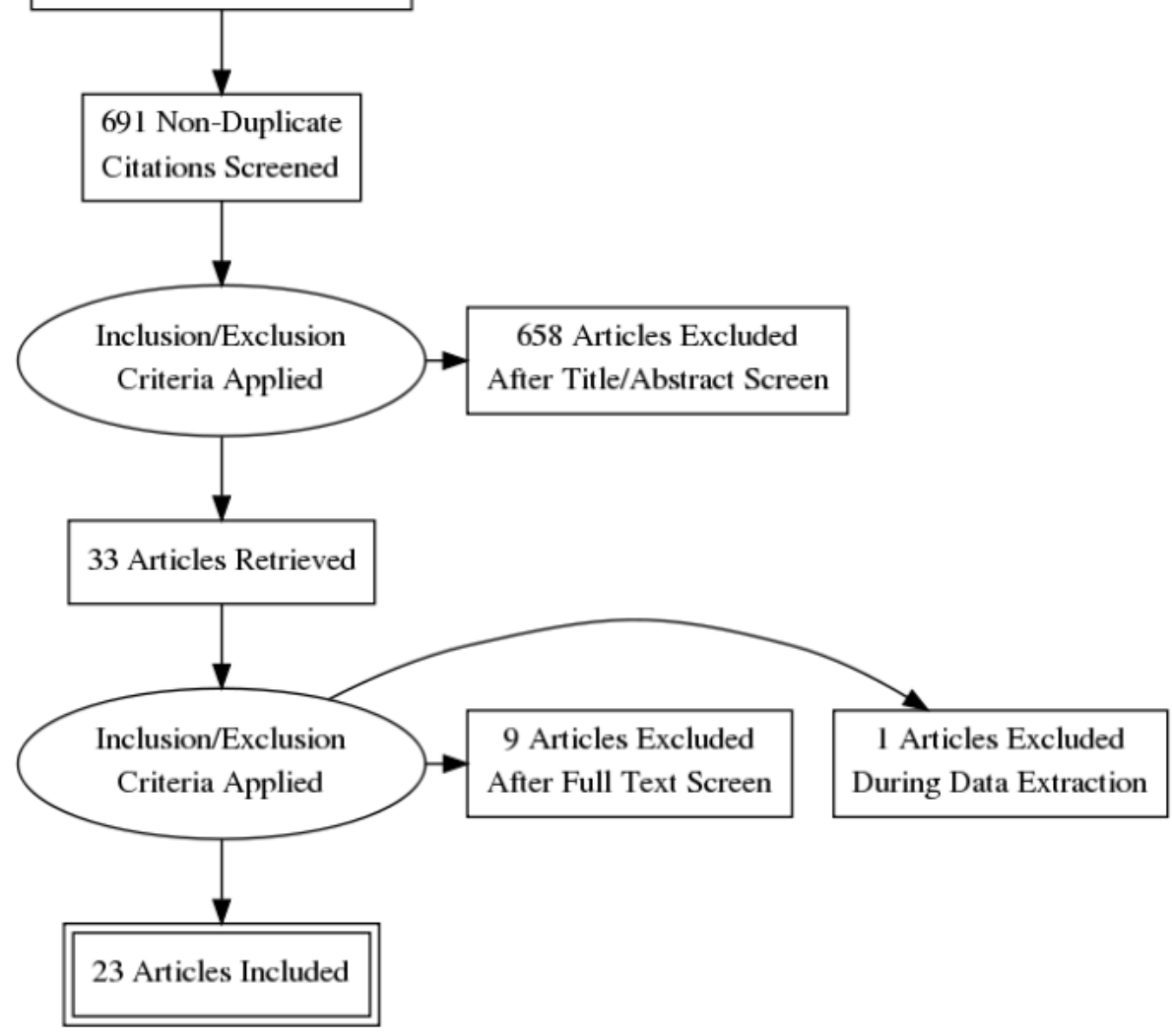

Figure 1

Figure shows the flow of papers through the study.

\section{Supplementary Files}

This is a list of supplementary files associated with this preprint. Click to download.

- GRIPP2shortform.docx 